\title{
El tratamiento de las neumonías no complicadas en la infancia con amoxicilina durante 3 días es tan efectivo como el de 5 días
}

Clinical efficacy of $\mathbf{3}$ days versus $\mathbf{5}$ days of oral amoxicillin for treatment of childhood pneumonia: a multicentre double-blind trial. PakistanMulticentre Amoxicillin Short Course Therapy (MASCOT) pneumonia study group. Lancet 2002; 360: 835-841.

\section{Objetivo}

Comparar la eficacia clínica del tratamiento con amoxicilina durante 3 o 5 días en las neumonías no severas de la infancia.

Diseño

Ensayo clínico multicentrico randomizado, doble ciego, placebo-control (con seguimiento hasta 14 días de finalizada la intervención). Lugar

Se realizó en siete hospitales ubicados en cinco ciudades de Pakistán. (Gilgit, Islamabat, Lahore, Multan y Rawalpindi).

\section{Pacientes}

Se reclutaron 2000 niños con edades entre 2 y 59 meses, que presentaban tos o dificultad respiratoria con aumento de su frecuencia (mayor $\mathrm{o}=\mathrm{a} 50 \mathrm{resp} / \mathrm{min}$. en chicos entre 2 y 11 meses, o mayor 0 = a $40 \mathrm{resp} / \mathrm{min}$. en chicos entre 12 y 59 meses) a los cuales se les diagnosticó neumonía no severa según los criterios de World Health Organization (WHO). Se excluyeron aquellos chicos que presentaban enfermedad crónica subyacente, historia de 3 o más episodios de dificultad respiratoria por asma bronquial, o aquellos que habían recibido antibiótico durante las 48 hs. previas al diagnóstico. Fueron excluidos 20 niños de la rama 3 días de tratamiento y 27 niños de la rama 5 días. Finalmente se incluyeron 980 y 973 niños, en cada rama respectivamente.

\section{Medición de resultados principales}

El análisis se realizó por intención de tratar. El punto final primario fue el fracaso del tratamiento, y el secundario fue la recaída de la enfermedad (definida por el desarrollo de algún signo de neumonía entre los días 6 y 14 después de normalizada la frecuencia respiratoria). La resolución clínica de la enfermedad se definió por el retorno de la frecuencia respiratoria al rango normal para el grupo etario evaluado.

\section{Resultados principales}

No se registraron diferencias significativas en los resultados clínicos de ambos regímenes terapéuticos.

El tratamiento falló en 209 (21\%) pacientes del grupo de 3 días y en $202(20 \%$ ) en el de 5 días ( diferencia 0,7\%; IC: - 1,8 a 3,2).

Las recaídas se observaron en 12 chicos (1\%) en el grupo de 3 días y $13(1 \%)$ en el grupo de 5 días (diferencia $0,1 \%$ IC 95 : - 0,6 a 0,8 ). En el análisis de regresión logística se apreció que la falla del tratamiento se asoció a incumplimiento $(4,5$ [2,9-7,0]; p menor 0,0001); edad entre 2-11 meses (1,7 [1,1-2,1], p menor 0,0001); frecuencia respiratoria mayor a $10 \mathrm{resp} / \mathrm{min}$. por encima del límite superior normal para la edad (1,4 [1,1-1,9], $p=0,004)$; duración de la enfermedad igual o superior a 3 días $(1,7[1,3-2,1], p=0,004)$ y vómito $(1,4[1,2-$ $2,0], p=0,009$ ).

Se reportaron efectos indeseables (diarrea, rash, vómito) de la medicación en $72(7 \%)$ chicos del grupo de 3 días de tratamiento, comparado con $76(8 \%)$ del grupo de 5 días en la primera evaluación, y $43(4 \%)$ y $57(6 \%)$ respectivamente en la segunda evaluación. La neumonía fue diagnosticada en el $14 \%$ de los chicos mediante radiografía, no habiendo diferencias entre ambos grupos $(1,5[0,8-$ $1,38], p=0,714)$. A475 chicos se les pudo realizar aspiración nasofaríngea con el posterior aislamiento del Virus Sincitial Respiratorio en $93(20 \%)$ chicos, sin diferencia entre los 2 grupos de tratamiento. De los 388 chicos a los que se les solicitó Proteína $C$ reactiva, el resultado fue positivo en 150 (39\%) el cual fue asociado con imagen radiográfica de neumonía ((3.6 (1.7-7,7), p menor 0,0001)).

\section{Conclusiones}

El tratamiento de las neumonías no severas (definidas por la WHO) con amoxicilina durante 3 días fue tan efectivo como el de 5 días. La causa más frecuente de falla fue la no-adherencia al mismo, cuyo porcentaje fue similar en ambos grupos.

Palabras claves

Amoxicilina. Neumonía. Tratamientos cortos. Ensayo randomizado. Doble ciego.

Fuente de financiamiento: Departament of Child and Adolesent. Health and Development, WHO, Geneva, of which Shamim Qazi is a medical officer.

\section{Comentario}

El presente trabajo demostró que se obtuvieron los mismos resultados (no se presentaron diferencias estadísticamente significativas) al tratar las neumonías no severas durante 3 o 5 días con amoxicilina (15 mg/ Kg/ cada $8 \mathrm{hs}$.), con un bajo porcentaje de fallas así como de recaídas en ambos. Cabe destacar la calidad metodológica en la asignación de los grupos y las similitudes entre ambos en relación con los efectos de la intervención. La neumonía fue diagnosticada siguiendo los criterios de la WHO, pudiéndose realizar solo en un pequeño porcentaje de chicos de ambos grupos (14\%) un estudio radiográfico para reconfirmar el diagnóstico; lo mismo sucede con la aspiración nasofaríngea y la Proteína $C$ reactiva. Resultaría sumamente interesante saber cual sería el resultado de dichos exámenes complementarios en el resto de los pacientes, para corroborar, de esta manera, cuantas neumonías fueron verdaderamente de etiología bacteriana. ${ }^{1,2,3 .}$

En el estudio se menciona que los pacientes que no respondieron a la terapéutica con amoxicilina, sí lo hicieron luego con alguno de los siguientes antibióticos: Cloranfenicol o cefixime vía oral, y algunos requirieron tratamiento inyectable con ampicilina/ penicilina benzatínica, cloxacilina, cefotaxime o cefuroxima.

No se menciona en el estudio la concentración inhibitoria mínima de los antibióticos como para evaluar si dichas dosis son extrapolables a nuestra población. ${ }^{4}$

Existe en la actualidad una muy limitada evidencia respecto a los tratamientos cortos (menores o iguales a 5 días) de las neumonías, lo cual hace que tengamos cierta precaución en relación con la validez externa de este tipo de estudios. ${ }^{5,6}$.

La mayoría de los trabajos publicados al respecto en los cuales se incluyó la amoxicilina como tratamiento (comparada con otros antibióticos), se realizaron con dosis algo superiores y los mismos duraron 7 o más días. ${ }^{7,8}$.

Existen pocos trabajos publicados con adecuada calidad metodológica que reafirmen los resultados del presente estudio por lo que seria necesario disponer de mayor información al respecto como para generalizar la aplicabilidad del presente trabajo.

\section{Dr. Javier Chiarpenello [ Médico Jefe de Centro de Salud № 29. Atención Primaria de Salud. Hospital Provincial de Rosario ]}

\section{Referencias}

1-Pneumonia in childhood: etiology and respouse to antimicrobial terapy. Ruuskanen O, Nohynek H, Ziegler T, et al. Eur J. Clin. Microbiol Infect Dis. 1992. Mar; 11 (3): $217-23$.

2-Appropriateness of antibiotic prescriptions in community-acquired acute pediatric respiratory infections in Spanish emergency rooms. Ochoa C, Inglada L, Eiros JM, et al. The Spanish Study Group on Antibiotic Treatments. Pediatr. Infect Dis J. 2001.Aug; 20 (8):751-8.

3-Magnitude of the problem of childhood pneumonia. Mulholland k. Lancet 1999; vol. 354:590-592.

4-Bacteriológical evidence of antibiotic failure in pneumococcal lower respiratory tract infections. Klugman K P. Eur. Respir. J. Suppl. 2002. Jul; 36: 3s-8s.

5-Clinical efficacy of co-trimoxazole versus amoxicillin twice daily for treatment of pneumonia: a randomized controlled clinical trial in Pakistan. Gatchup Study Group. Arch. Dis Child 2002. Feb; 86 (2):113-8.

6-Clinical response to two days of oral amoxicillin in children with non-severe pneumonia. Awasthi S. Indian Pediatr. 2000. Mar; 37 (3):301-6.

7-Pneumonia. Kabra SK, Singhal T, Lodka R. Indian J. Pediatr. 2001. Jul; 68 Suppl. 3: 519-23.

7-Pneumonia. Kabra SK, Singhal T, Lodka R. Indian J. Pediatr. 2001. Jul; 68 Suppl. 3: 519-23. 1998. Oct; 14 (5): 338-41. 